\title{
Linx
}

Revue des linguistes de l'université Paris X Nanterre

$75 \mid 2017$

Imaginaires de la ponctuation. Ordre et inquiétude du discours

\section{Qu'est-ce que la « valeur expressive » en grammaire ? Le cas de la ponctuation}

Éric Bordas

\section{(2) OpenEdition}

\section{Journals}

Édition électronique

URL : http://journals.openedition.org/linx/1908

DOI : $10.4000 /$ linx.1908

ISSN : 2118-9692

Éditeur

Presses universitaires de Paris Nanterre

Édition imprimée

Date de publication : 22 décembre 2017

Pagination : 127-144

ISBN : 978-2-84016-308-4

ISSN : 0246-8743

Référence électronique

Éric Bordas, «Qu'est-ce que la « valeur expressive » en grammaire ? Le cas de la ponctuation », Linx

[En ligne], 75 | 2017, mis en ligne le 23 novembre 2018, consulté le 19 avril 2019. URL : http://

journals.openedition.org/linx/1908; DOI : 10.4000/linx.1908

Ce document a été généré automatiquement le 19 avril 2019

Département de Sciences du langage, Université Paris Ouest 


\title{
Qu'est-ce que la « valeur expressive » en grammaire? Le cas de la ponctuation
}

\author{
Éric Bordas
}

1 La présente étude est inspirée par deux questions générales qui peuvent sembler étrangères l'une à l'autre mais dont on a l'intuition qu'elles sont deux variables théoriques d'une même interrogation: 1) Qu'est-ce que cette mystérieuse "valeur expressive» que les grammairiens évoquent parfois pour commenter certaines réalisations linguistiques du discours ? 2) La ponctuation relève-t-elle de la grammaire, c'est-à-dire est-elle un objet apte à entrer, comme la morphologie ou la syntaxe, dans un discours descriptif normé des formes et des usages généraux de la langue?

\section{Les grammairiens contemporains et la « valeur expressive »}

2 Les années 1990 ont vu une multiplication en France de nouvelles grammaires, publiées et intitulées comme telles, principalement à destination des étudiants de Lettres et de leurs enseignants, synthèses heureuses d'une conception générale qui restait à repères référentiels grammairiens (le plan) et de connaissances et approches linguistiques (analyses): Gardes-Tamine (1990), Charaudeau (1992), Le Goffic (1993), Riegel, Pellat, Rioul (1994), puis Wilmet (1997). Toutes se référent, quoique très ponctuellement et discrètement ${ }^{1}$, à la notion d'expressivité pour commenter tel ou tel phénomène ${ }^{2}$.

Ce peut être sans précaution particulière, par exemple pour opposer deux pratiques accentuelles: «En français, il faut distinguer deux types d'accent, un accent dit interne, non emphatique, et un accent dit externe ou emphatique lié à des facteurs expressifs et intellectuels alors que le premier est purement linguistique » (Gardes-Tamine, 1990: I, 20; je souligne) $)^{3}$. Mais ce peut être aussi avec une manifeste insatisfaction proche d'une mauvaise conscience (?), précisément marquée par des signes typographiques comme des 
guillemets de détachement à valeur de connotation autonymique pour indiquer la nature problématique du mot utilisé faute de mieux. Ainsi de Pierre Le Goffic analysant le tour interrogatif en Est-ce que $P$ ? originellement à valeur causale ou emphatique ${ }^{4}$ et concluant (1993 : 102) : « la valeur “expressive” du tour s'est estompée, et est-ce que n'est plus senti que comme un instrument d'interrogation ».

4 Aussi cursifs soient-ils, et surtout opposés stylistiquement avec cet emploi du mot en usage ou en mention, ces deux premiers exemples d'une référence à la notion d'expressivité, pour commenter un tour syntaxique (Le Goffic) ou un phénomène prosodique (Gardes-Tamine), se rejoignent dans l'identification implicite de la nature particulière du cas envisagé, lequel s'oppose à la règle générale qui serait le propre de la grammaire. Joëlle Gardes-Tamine oppose un accent qui serait «purement ( sic) linguistique " parce qu'interne et propre à la nature même de la langue française à un accent « emphatique lié à des facteurs expressifs et intellectuels ». On ne saurait être plus clair, ni plus schématique : d'un côté la langue (générale), prétendument non marquée ; de l'autre, le discours (particulier), identifiable par des marqueurs externes relevant de la personnalité propre du sujet. Même si son propre constat lui déplait, de toute évidence, Pierre Le Goffic raisonne de la même façon, en concluant par une restriction pour juger que le tour envisagé s'est départi de sa valeur originale d'intensification, avec accusation implicite $^{5}$, pour ne plus être qu'un marqueur linguistique d'interrogation : il y a bien une gradation et l'on devine le grammairien de la phrase heureux d'être débarrassé d'éléments discursifs particuliers mal intégrables dans sa démonstration descriptive générale.

Ces deux exemples découvrent ce qui est la règle de la référence à l'expressivité dans les grammaires : le cas particulier où s'arrête la langue de tous et où commence le discours du sujet individuel dans son histoire. Les occasions ne manqueront pas, évidemment. Pour commenter l'« axe principal» de l'ordre des mots en français, Marc Wilmet (1997: 533-8) distingue les "facteurs grammaticaux» qui correspondent aux cas standards linéaires et les «facteurs expressifs » que représentent, selon lui, les focalisations par dislocation qui propulsent les objets ou les attributs devant le verbe ${ }^{6}$. De même, travaillant sur la catégorie grammaticalement sinon linguistiquement problématique de la séquence averbale, dite "phrase nominale», Martin Riegel et alii (1994: 457) concluent: «la phrase nominale pourra, selon son rapport avec l'énonciation, prendre une valeur générale ou au contraire particulière. Dans ce cas, elle manifeste souvent une plus grande expressivité que la phrase canonique ». Et ils ajoutent la référence attendue qui résume l'idée: "Selon G. Guillaume, quand l'expression grammaticale se réduit, l'expressivité croit, l'expressivité maximale étant illustrée par les interjections» (je souligne) $)^{7}$.

\section{Expression grammaticale vs expressivité (stylistique)?}

6 Il conviendrait ainsi de distinguer avec soin l'expressivité particularisante et individualisante de l'expression générale commune. L'expression serait une simple production linguistique dont la grammaire décrirait les réalisations normalisées, proche synonyme du signifiant ou, par métonymie, de son contenu; l'expressivité serait l'appropriation singulière de cette expression sur un axe discursif à approfondir (ou non). On y revient: il est difficile de ne pas retrouver là la dichotomie fondatrice langue/ 
discours. L'expressivité est dès lors la marque et la mesure d'un sujet individuel dans la langue, qui inscrit le sens produit de l'énoncé dans l'histoire de sa production ${ }^{8}$. Ce qui, à partir de là, notons-le avant d'y revenir, pose la question de la valeur (historique ?) de cet énoncé dans cette production temporalisée - cette "valeur» que Pierre Le Goffic invoquait pour thématiser la réalisation pragmatique constatée qui n'est pas une fonction.

7 En somme, et dans cette perspective, il n'est pas exagéré d'estimer que l'expressivité serait la réalisation d'un style, et son apparition marquerait la fin du champ de la grammaire, science du général, pour saluer le début de celui de la stylistique, science du particulier $^{9}$ ? On sait que c'était la thèse fondatrice de Bally qui justifiait, pour lui, la création de la stylistique comme discipline autonome : le langage sert à exprimer des idées et des sentiments, ce qui signifie « extérioriser toute la partie intellectuelle de notre être pensant " et toute sa partie "affective " (Bally, 1951: I, 5-6). On mesure l'importance fondamentale du préfixe de mouvement extériorisant ex-: l'expressivité est l'activité qui fait émerger à la conscience par le langage le monde obscur de l'esprit et de l'affect ; elle correspond à une dynamique individualisante ascendante, proche d'une maïeutique, qui fait passer de l'informulable à l'exprimé.

Bally illustre son idée par une progression d'exemples, partant d'un banal «Je suis étonné de vous voir ici", dont il précise qu'il faut, "pour que cette forme de pensée soit exclusivement intellectuelle (sic), que l'intonation, l'inflexion de la voix soit assez inexpressive pour ne révéler aucune trace d'élément affectif ou émotif »-ce qui serait, du reste, « un cas extrême » (1951: 7). Il décrit ensuite la progression expressive, partant de cette base neutre pour évoluer vers des formes de plus en plus marquées : «Imaginez maintenant une proportion toujours plus grande d'émotion dans le fait de pensée, vous obtiendrez une gradation parallèle dans l'expression : "Tiens! Vous êtes ici ?" - "Comment ! Vous ici ?" - "Vous!", jusqu'à ce qu'enfin l'émotion, ne trouvant plus dans les mots d'expression adéquate, s'extériorise dans une exclamation pure, telle que : “oh!" » - et Bally précise que "cette interjection » trouvera une réalisation expressive encore plus forte «si elle est soulignée d'une intonation susceptible de marquer toute l'intensité de l'émotion » (ibid. : 7-8).

On voit que l'idée rappelée par Martin Riegel et alii citée précédemment et par eux attribuée à Guillaume, "quand l'expression grammaticale se réduit, l'expressivité croit, l'expressivité maximale étant illustrée par les interjections", correspond exactement, mot à mot, aussi et surtout à la pensée de Bally ${ }^{10}$. Précisément parce que c'est une pensée stylistique de la langue et non une pensée grammairienne. Et c'est une pensée stylistique parce que, il faut bien le reconnaître, elle procède par pensée d'un écart fondateur à partir duquel se mesurent des niveaux d'expressivité :

Degré zéro (qui correspond pour Bally à une inexpressivité de la locution) : une phrase standard avec GN sujet + GV prédicat - Je suis étonné de vous voir ici.

11 Degré 1 de l'expressivité marquée révélant le monde propre du locuteur, sa personnalité, son caractère: une phrase standard GN + GV, mais assortie d'incidences exclamatives exogènes complémentaires (une intonation forte en deux temps + un impératif lexicalisé en interjection) - Tiens! Vous êtes ici?

Degré 2 de cette même expressivité : une séquence averbale, mais articulée en deux temps complémentaires avec protase et apodose-Comment! Vous ici? 

ponctuation marquée par les quelques signes typographiques d'usage fort (? et!), mais tout autant par la diction textuelle toujours plus resserrée et concentrée des énoncés. Et ce n'est en rien le fait d'un hasard.

17 Absolument liée à la production textuelle d'une séquence dont elle marque les différentes étapes linéaires dans la durée, la ponctuation n'existe pas sans affirmation d'un sujet singulier originel: on peut même dire que sa première fonction est de rappeler l'existence de ce sujet. L'expressivité étant définie par Bally comme toute extériorisation langagière de la pensée ou émotivité intime du locuteur, la ponctuation ne saurait être autre chose que l'expression formelle d'une expressivité originale perçue dans une scansion, une diction.

C'est pourquoi son appartenance au regard globalisant de la grammaire ne peut que rester très incertaine, à moins de l'envisager non comme possible matière scientifique d'études linguistiques, mais comme objet de prescriptions, comme pour un traité, de Damourette (1939) à Drillon (1991), qui assignerait et consignerait les bons et les mauvais usages $^{11}$ : les règles de ponctuation - laquelle rejoint ainsi l'orthographe, dont le respect fut longtemps la principale raison d'être des grammaires ${ }^{12}$. Forme même de la diction originale d'un sujet singulier dans l'histoire (définie comme temps vécu), perceptible dans et par les pulsations dynamiques du discours produit, la ponctuation est, comme le rythme dont elle n'est du reste qu'une réalisation marquée ${ }^{13}$, un niveau de base de réalisation du style comme expérience de l'individuation par l'individualisation.

Cela posé, il convient de rappeler une évidence: toutes les marques écrites de ponctuation n'ont pas a priori le même potentiel expressif - et s'il est bien un objet pour lequel l'opposition artificielle écrit/oral est bien un redoutable piège, c'est assurément la ponctuation: en dépit de la caution de Beauzée et de toute la tradition rhétorico- 
grammairienne, la ponctuation de l'écrit n'a tout simplement rien à voir avec la ponctuation à l'oral (qui n'est pas ponctuation de l'oral) ${ }^{14}$. Autant le point d'exclamation est entièrement un signe fort d'expressivité, à sens variable mais du reste assez limité, marqueur avant tout de distanciation par rapport à l'énoncé qui le précède, autant les trois points de suspension ont un riche potentiel en matière de sous-entendu, de suggestion, autant guillemets et italiques sont essentiels aux ressources de la polyphonie, autant les autres signes de ponctuation peuvent paraitre d'emploi plus transparent, plus banalement utilitaire, simples reconductions des grands repères syntaxiques et modaux d'une « phrase » ${ }^{15}$.

Mais, précisément, les petits exemples tout simples de Bally ont montré que les ressources stylistiques d'une ponctuation expressive ne se réduisaient en rien aux quelques signes typographiques répertoriés par les traités d'inspiration grammairienne : d'un point de vue stylistique, la ponctuation la plus subtilement expressive d'un énoncé est peut-être tout entière en-dehors de l'arsenal des artificiels marqueurs exogènes d'énonciation, dans la forme même de la phrase et surtout dans la liaison des phrases entre elles ${ }^{16}$.

\section{Des ressources stylistiques de l'expressivité énonciative : surexpression et laconisme de/dans la ponctuation}

21 Commençons donc par le point d'exclamation, dont la raison d'être, entièrement, est d'indiquer aussi clairement que possible une expressivité de registre variable. Prétendue transcription graphique d'un ton oral, cette variable prosodique qui précise le sens de l'énoncé sous la signification de l'énonciation, le point d'exclamation désigne rétroactivement la séquence qui le précède comme matière à critique, critique valorisante (admiration) ou dévalorisante (désapprobation), mais mesure d'une valeur présentée comme discutable. Tout point d'exclamation est en soi un volontaire signe d'expressivité procédant d'une intention d'orientation sémantique de la réception du message produit. Or il existe un moyen très simple de renforcer l'expressivité pour outrer la représentation du sentiment qui la supporte, c'est de multiplier les points d'exclamation. Un diariste aussi véhément et emporté que Léon Bloy raffole du procédé et l'on ne compte plus, dans son Journal, les séquences commentées et conclues par des séries de trois, quatre ou cinq points d'exclamation rageurs et indignés selon des combinaisons variées ${ }^{17}$ :

[1] J'estime que votre offre de cent cinquante francs! voyage compris!!! à un écrivain qu'on suppose vaincu par la misère est insultante pour cet écrivain et déshonorante pour la Belgique. ${ }^{18}[2]$ Ce successeur d'Aaron m'affirme qu'IL Y A DU BON DANS TOUTES LES RELIGIONS!!!19 [3] Le Mercure publie un article de de Gourmont où il est dit que Jésus a manqué de logique dans ses paroles !!!!!20 [3'] Ce pauvre F. me reproche surtout de ne pas écrire comme tout le monde!!! C'est effrayant, c'est à pleurer. ${ }^{21}[4]$ Cet incontestable voyou parle même de la Charité chrétienne (!!!!) qui m'interdit les injures. ${ }^{22}\left[4^{\prime}\right]$ Lu, dans Le Journal, une interview, par correspondance, de plusieurs personnages importants à qui on demande ce qu'ils pensent de la mort (!!!) ${ }^{23}$ [4"] Un séducteur n'a pas le droit (!) d'épouser une fille séduite, si un enfant intervient $(! ! ?)^{24}$

Le premier exemple insère un premier point d'exclamation après un GP et avant un autre GP qui vient compléter l'actance prédiquée, et redouble cette première ponctuation forte 
d'un GN non actualisé et détaché, lui-même suivi de trois points d'exclamation: la ponctuation expressive n'est pas isolée en fin de phrase, mais intervient en cours d'énonciation pour modaliser le sentiment associé à deux référents: le prix, et la condition circonstancielle de ce prix. C'est là un cas relativement rare, le point d'exclamation se rencontrant le plus souvent en fin de séquence, modalisant l'ensemble de l'énonciation et non tel ou tel mot de l'énoncé. Dans ce cas, on ne peut pas ne pas comprendre la gradation dans les sentiments montrés par l'artifice typographique: l'indignation devant la somme allouée, marquée par un point unique, et renforcée par la précision de la condition, ce qui entraîne un triplement du point.

La quasi totalité des autres exemples placent le ou les points en fin de phrase, sauf en [4] et [4"] où l'on retrouve le point en milieu de phrase après le GN sensible ainsi désigné anaphoriquement par le point d'exclamation, mais alors isolé entre parenthèses (et triplé en [4]). Mais le seul point d'exclamation, même triplé, ne semble pas suffire à la colère de Bloy. En [2] et [3] il s'appuie, pour renforcer l'affirmation de l'axiologie, sur les ressources de la typographie pour réaliser un effet de polyphonie en dramatisant la représentation d'une citation : par les petites capitales en [2] ${ }^{25}$, par les italiques en [3]. Bloy va jusqu'à cinq points d'exclamation en [3] pour commenter les citations rapportées, ce qui contraste éloquemment en [3'] avec l'absence de toute signe exogène dans la séquence qui suit : «C'est effrayant, c'est à pleurer ». De même, les exemples de la série [4] jouent, pour leur part, d'une autre disponibilité typographique, la parenthèse, pour visualiser le sentiment suggéré par les points d'exclamation, ce qui isole mieux qu'en [1] la marque axiologique, résolument détachée du GN par la mention duquel la violence est provoquée. Enfin, on remarque en [4"] dans ce système de parenthèse la combinaison d'un point d'interrogation à deux points d'exclamation dont la fonction est très clairement de remettre en question la simple possibilité d'exister de l'objet évoqué, sa pertinence conceptuelle.

Bloy multiplie donc les marqueurs exogènes typographiques dans sa phrase, à l'intérieur de la séquence comme en conclusion, afin de représenter visuellement son affect. La logique est banalement quantitative: plus le nombre de signes sera grand, plus efficace sera censée être la représentation de l'émotion ${ }^{26}$. Nous avons là un exemple très simple d'expressivité stylistique posée et construite par la typographie qui répond à l'une de ses conceptions répandues : l'indication de nuances émotives permettant à un locuteur de suggérer, autant que possible, ses sentiments les plus sincères, comme le feraient immanquablement à l'oral son intonation, son timbre de voix, son visage, ses gestes. Nul n'a mieux résumé cette idée qu'Henri Meschonnic en une dense formule: "La ponctuation est la part visible de l'oralité » (2000:289) - que l'on nuancerait toutefois volontiers en : «La ponctuation est la part sensible de la temporalité » : graphie du temps (de l'émotion) dans la subjectivation de l'écrivant. Le langage est instrument de représentation, mise en forme par des signés codés par l'usage et la norme d'un référent, en l'occurrence émotif, extérieur à lui-même, et l'expressivité est l'une des mesures de sa performance stylistique.

À cette surexpression par multiplication des signes et des marqueurs incidents externes s'oppose stylistiquement le laconisme de l'effacement. La ponctuation n'est plus alors dans les points d'exclamation, mais dans la liaison des phrases par les blancs. Ainsi, chez le même Bloy, la tonitruance grimaçante peut faire place à un détachement lui aussi fort expressif, mais d'une discrétion telle qu'il risque presque de passer inaperçu : 
[5] Il se dit anarchiste et littérateur, mais dans quelle langue, et en arborant quelle caroncule de dindon! J'ai voulu savoir si j'avais affaire à un imbécile. Je suis fixé. C'est un parfait imbécile. ${ }^{27}$ [6] Ah! que Dieu fut bon pour ses pauvres, ce jour-là ! / C'était le 13 novembre. On venait de voir disparaître les derniers centimes, on pouvait être jeté dans la rue, le lendemain; nos meubles, en compagnie de ma bibliothèque et de mes papiers, flottaient sur la mer du Nord. ${ }^{28}$ être allé à la ligne, Bloy déploie une série de quatre phrases simples, elles aussi reliées implicitement par asyndète et procédant par approfondissement et resserrement du cadrage textuel et du champ visuel dans une représentation narrative : 1) cadre général ( C'était le 13 novembre »);2) arrière-plan général (« On venait de voir disparaître les derniers centimes »);3) indication du contexte particulier (« on pouvait être jeté dans la rue, le lendemain ») ; 4) introduction du sujet singulier dans un pluriel d'identification («nos meubles [...] flottaient sur la mer du Nord »). Les faits, rien que les faits, mais de cette énonciation dégraissée de toute l'axiologie dont la séquence initiale était chargée se dégage une progression émotive qui est le récit d'un miracle ${ }^{30}$. La dramatisation de l'énonciation se construit également dans la ponctuation textuelle par deux paragraphes complémentaires, le premier thématisant, le second prédicant ${ }^{31}:$ l'ensemble découvre une ponctuation d'abord "trace des processus de linéarisation " de la représentation discursive (de l'émotion) ainsi devenue «lieu privilégié d'accomplissement de la planification » stylistique du texte par le sujet écrivant (Passerault, 1991 : 90-1).

\section{Ponctèmes exogènes, ponctèmes $\emptyset$ endogènes}

La différence avec la surcharge des signes de ponctuation expressifs de la série [1-4] est radicale : à un style véhément s'oppose un style laconique, comme à des vociférations furieuses s'opposerait un silence, ironiquement résigné en [5], recueilli en [6]. Pourtant, dans ces deux exemples aussi nous avons une prose soumise à une surexpression émotive, une prose qui affiche une expressivité sensible qui est tout son propos. Il serait naïf de penser que l'expressivité est plus forte en [1-4], avec la grêle de points d'exclamation qui viennent s'incruster en rafales dans le texte du discours, qu'en [5-6], avec cette 
dominante d'une asyndète simple: le choix de l'oxymore "parfait imbécile» ou de l'image « jeté dans la rue » montre bien que la violence est là. Mais Bloy ne la soumet pas au même régime d'énonciation, et c'est dans la modification de la ponctuation que la différence est la plus sensible.

30 Aux ponctèmes exogènes de la première série répondent ce que l'on appellera, faute de mieux et en pensant à la complémentarité déterminant/déterminant $ø$, les ponctèmes $\varnothing$ endogènes de la seconde série: liaison des phrases les unes après les autres, cohésion isotopique des temps et des personnes par anaphore et répétition, division du texte en paragraphes complémentaires. La ponctuation n'est plus le fait de petits symboles typographiques plérémiques ajoutés dans la prose dans une activité de complémentation discursive pour représenter une émotion qui double le sens premier de l'énoncé et produit ainsi une expressivité figurative: elle est interne à l'énonciation, donc non traduisible en signes conventionnels externes, elle se confond absolument avec le discours et n'est pas décomposable en unités complémentaires, elle relève du « système silencieux » (Rault, 2014b) qui (et que) porte également une certaine pratique discursive de ponctuation ${ }^{32}$.

31 La diction de la ponctuation se rapproche ainsi à son maximum de la temporalité du discours : temps représenté et temps représentant. Cette ponctuation correspond, par exemple, aux heures ou aux minutes qui viennent ponctuer, dit-on, par le sentiment soudain de leur présence une journée d'attente. Ce pourquoi elle est une forme du rythme, cette inscription sensible du sujet dans son discours. Elle est peut-être, à premier abord, moins démonstrativement expressive que la ponctuation par signes exogènes, dont le repérage est immanquable et le décodage aisé, mais elle n'en propose pas moins des effets de valorisation pragmatique de l'énonciation qui la contient.

On prendra garde de ne pas opposer ces deux pratiques stylistiques comme relevant d'une opposition de type explicite/implicite. Pour ne pas avoir de réalisations originales et appropriées par identification normée, les ponctèmes $\varnothing$ endogènes ne sont pas des indices d'un sous-entendu, ni moins encore une forme d'ellipse sollicitant le rétablissement d'une unité qui viendrait tout expliquer. Encore une fois, le contenu émotif des exemples [5] et [6] n'a rien d'implicite, et la progression sensible qui est décrite dans leur ponctuation sollicite moins un sens caché que la valeur d'une posture distancée. Le choix d'une ponctuation endogène est, certes, celui d'une discrétion stylistique, mais ce n'est pas là une question sémantique. Ce qui démontre, s'il en était besoin, que la sémiotique de la ponctuation, souvent exagérée du fait d'une fixation, par réflexe grammairien, sur les quelques marques typographiques qui lui sont associées, reste en attente d'une sémantisation originale que le sujet investira de son histoire propre.

Peut-on dès lors et pour réunir les deux questions initiales de cette étude, questions complémentaires, envisager une grammaire de l'expressivité posée et représentée par l'énonciation dans les manifestations textuelles de ce que l'on appelle "ponctuation »? Oui, s'il s'agit d'une grammaire stylistique, c'est-à-dire une grammaire du singulier qui s'attache à décrire tout ce qu'un sujet individuel peut faire passer de lui-même par la langue dans un énoncé et qui travaille sur la valeur comme performance dans ce vaste régulateur discursif qu'est le style - mais serait-ce alors encore une grammaire? Dans cette perspective, la ponctuation ne peut que jouer un rôle majeur, se confondant entièrement avec l'énonciation, phénomène induit et produit par l'historicité fondatrice d'un sujet s'exprimant dans le temps de son discours: loin d'être un complément d'information, elle découvrirait même la base de la diction textuelle sans laquelle il n'y 
aurait pas de style. Mais, référence fortement sémiotisée avec son arsenal de petites unités typographiques qui l'incarnent et la résument dans un certain imaginaire linguistique, la ponctuation doit être pensée aussi dans l'absence de ces signes, dans ce qui n'est en rien une ellipse, mais la forme d'un style de retrait, de distanciation et d'absence. Car la ponctuation, unité rythmique par définition, est moins un système de signes répertoriables qu'un aspect, parmi d'autres, d'une expérience de discours dont une incertaine expressivité assure, seule, la lisibilité.

\section{BIBLIOGRAPHIE}

ANIS, J., 2004, « Les linguistes français et la ponctuation », dans S. Pétillon (dir.), L'Information grammaticale, 102, La Ponctuation, p. 5-10.

APOTHÉLOZ, D., COMBETTES, B., \& NEVEU, F. (dir.), 2009, Les Linguistiques du détachement, Peter Lang, Bern.

ARABYAN, M., 1994, Le Paragraphe narratif. Étude typographique et linguistique de la ponctuation textuelle dans les récits classiques et modernes, L'Harmattan, Paris.

BACHA, J., 2000, L'Exclamation. Approche syntaxique et sémantique d'une modalité énonciative, L'Harmattan, Paris-Montréal.

BALLY, C., 1951, Traité de stylistique française, Georg \& Klincksieck, Genève-Paris.

BESSONNAT, D. (dir.), 1991, Pratiques, 70, La Ponctuation, Metz.

BOONE, A., \& JOLY, A., 2004, Dictionnaire terminologique de la systématique du langage, L'Harmattan, Paris-Budapest-Torino.

CHARAUDEAU, P., 1992, Grammaire du sens et de l'expression, Hachette, Paris.

DAMOURETTE, J., 1939, Traité moderne de ponctuation, Larousse, Paris.

DEFAYS, J.-M., ROSIER, L., \& TILKIN, F. (dir.), 1998, À qui appartient la ponctuation ?, Bruxelles, Duculot.

DENIS, D., \& SANCIER-CHATEAU, A., 1994, Grammaire du français, Librairie Générale Française, Paris.

DRILLON, J., 1991, Traité de la ponctuation française, Gallimard, Paris.

DÜRRENMATT, J., 1998, Bien coupé mal cousu. De la ponctuation et de la division du texte romantique, PUV, Saint-Denis.

DÜRRENMATT, J. (dir.), 2000, La Licorne, 52, La Ponctuation, Poitiers.

DÜRRENMATT, J., 2001, Le Vertige du vague. Les Romantiques face à l'ambiguïté, Kimé, Paris.

DÜRRENMATT, J., 2015, La Ponctuation en français, Ophrys, Paris.

GARDES-TAMINE, J., 1990, La Grammaire, Armand-Colin, Paris.

GAUTIER, L., \& MONNERET, P. (dir.), 2010, La Fonction expressive. Volume 2, Presses universitaires de Franche-Comté, Besançon. 
JAFFRÉ, J.-P., 1991, « La ponctuation du français : études linguistiques contemporaines », dans D. Bessonnat (dir.), op. cit., p. 61-83.

KUYUMCUYAN, A., 2009, « Un problème de ponctuation ? Des compléments après le point ", dans D. Apothéloz et al. (dir.), op. cit., p. 317-32.

LEGALLOIS, D., \& FRANÇOIS, J., « Définition et illustration de la notion d'expressivité en linguistique », CRISCO, Université de Caen - disponible sur internet.

LE GOFFIC, P., 1993, Grammaire de la phrase française, Hachette, Paris.

MESCHONNIC, H., 2000, « La ponctuation, graphie du temps et de la voix », dans J. Dürrenmatt (dir.), op. cit., p. 289-93.

MONNERET, P., 2010, «Expressivité et image : retour sur la conception guillaumienne de l'expressivité », dans L. Gautier \& Ph. Monneret (dir.), op. cit., p. 19-35.

PASSERAULT, J.-M., 1991, « La ponctuation. Recherches en psychologie du langage », dans D. Bessonnat (dir.), op. cit., p. 85-104.

PÉTILLON, S. (dir.), 2004, L'Information grammaticale, 102, La Ponctuation, Paris.

POPIN, J., 1998, La Ponctuation, Nathan, Paris.

RAULT, J., 2014a, «De la "pause” à la "valeur” en langue : grammaticalisation des signes de ponctuation ", Congrès Mondial de Linguistique Française, Berlin - disponible sur internet.

RAULT, J., 2014b, « La ponctuation : problématiques linguistiques », Le français aujourd'hui, 187, Paris, p. 9-18

RIEGEL, M., PELLAT, J.-C., \& RIOUL, R., 1994, Grammaire méthodique du français, PUF, Paris.

ROSIER, L., 1998, « Discours grammatical et ponctuation : l'exemple du discours rapporté », dans J.-M. Defays et al. (dir.), op. cit., p. 353-64.

SENSINE, H., 1930, La Ponctuation en français, avec soixante dictées-exercices dans le texte, Payot, Paris. SERÇA, I., 2004, « La ponctuation : petit tour d'horizon », dans S. Pétillon (dir.), op. cit., p. 11-17. SERÇA, I., 2012, Esthétique de la ponctuation, Gallimard, Paris.

VÉDÉNINA, L., 1989, Pertinence linguistique de la présentation typographique, Peeters/Selaf, Paris. WILMET, M., 1997, Grammaire critique du français, Hachette, Paris.

\section{NOTES}

1. À l'exception de P. Charaudeau qui prétend en faire son objet propre : voir infra. 2. Pour une synthèse sur la question de l'expressivité en linguistique, voir Legallois \& François (s. d.) et Gautier \& Monneret (2010) qui remarquent : « les sciences du langage paraissent ne pas pouvoir se passer de ce concept tout en demeurant inaptes à en fixer une définition durablement heureuse » (ibid. : 9). Évidemment, le fait que les ouvrages ici considérés se présentent comme (et soient incontestablement telles) des grammaires est de la plus haute importance, voir infra.

3. L'auteur précise : «Les fonctions de cet accent sont multiples. C'est en premier lieu une fonction démarcative, puisque, frappant toujours la dernière syllabe d'un groupe, il en signale une des frontières. [...] C'est en second lieu une fonction contrastive ou culminative, qui se traduit par la mise en relief de la syllabe accentuée par rapport aux 
syllabes atones qui l'entourent. Enfin, et c'est peut-être là son rôle essentiel, l'accent joue un rôle dans l'intonation [...]. L'accent externe ou emphatique est également fixe, mais frappe cette fois la première syllabe d'un mot. Il est lié à une extension expressive » (ibid. : 21-2).

4. Exemple : Est-ce donc que vous m'en voulez?

5. Accusation injonctive renforcée dans l'exemple cité ci-dessus (n.) par la conjonction donc, mais encore plus nette dans les deux autres exemples donnés par l'auteur : Serait-ce que vous m'en voulez? Ne serait-ce pas plutôt que vous n'osez pas le dire?

6. Exemples : Hitler, connais pas. Les épinards en branche, moi, je déteste.

7. L'expressivité est un grand concept guillaumien. Si, dans une perspective opérative expression désigne le processus même de l'énonciation et renvoie à l'idée d'une grammaticalité normative, expressivité renvoie à l'ensemble des moyens verbaux et non verbaux dont dispose le sujet pour manifester sa subjectivité en mettant en relief telle ou telle partie de l'énoncé. L'expressivité guillaumienne est notoirement très proche de la fonction expressive de Jakobson. « Il apparaît à l'évidence que, dans le modèle guillaumien, l'expressivité n'est pas une épiphénomène lié aux variations infinies du discours, mais que c'est une donnée fondamentalement inscrites dans les potentialités systématiques. Les implications théoriques [...] sont importantes : la langue ne devrait pas être considérée restrictivement comme une simple "compétence" d'expression (une compétence "linguistique" stricto sensu), mais aussi comme une compétence expressive, qu'on peut sans abus qualifier de "pragmatique" »- A. Joly, in A. Boone \& A. Joly (2004 : 177). Sur la conception guillaumienne de l'expressivité, voir Monneret (2010) et infra. 8. Ce que dit exactement, dans ses intentions liminaires, P. Charaudeau prétendant proposer une Grammaire du sens et de l'expression (1992), soit une grammaire qui s'intéresse à décrire les faits de langage en fonction : «- des intentions du sujet parlant qu'ils sont susceptibles d'exprimer, ce qui exige que les catégories de la langue soient regroupées autour de ces intentions ; - des enjeux communicatifs qu'ils révèlent, ce qui exige que les différents systèmes de la langue soient traités du point de vue du sens ; - des effets de discours qu'ils peuvent produire, ce qui exige que soient passés en revue les différents types d'usage vivant de la langue, et pas seulement les usages littéraires » (ibid. : 4). 9. Dans une autre grammaire contemporaine qui peut aussi entrer dans notre corpus, Denis \& Sancier-Chateau (1994), on voit les auteurs éviter l'emploi de l'adjectif expressif pour utiliser à la place stylistique qui semble, dans ce contexte de commentaire d'usages, fonctionner comme un synonyme. Ainsi, à propos de la place variable de l'adjectif épithète, D. Denis \& A. Sancier-Chateau (ibid. : 13), après avoir énuméré les « facteurs formels » et les «facteurs sémantiques » des positions, présentent les « facteurs stylistiques » : « Lorsque la variation de place n'entraîne pas de conséquence sur le plan de l'interprétation, elle peut relever d'une volonté de marquage stylistique. Ainsi l'adjectif antéposé prendra volontiers une valeur subjective, propre à traduire l'appréciation ou l'affectivité de l'énonciateur. »Cette « traduction » de l'affectivité de l'énonciateur ressemble furieusement à l'expressivité invoquée par leurs collègues. 10. Le rapprochement entre Guillaume et Bally autour de cette idée d'expressivité a été étudié par Ph. Monneret (2010), qui cite le même extrait de Bally que ci-dessus mais qui insiste, à juste titre, sur la pomme de discorde que constitue entre les deux penseurs la notion d'affectivité pour expliquer l'expressivité. Là où Bally en fait un principe général, Guillaume réplique que « l'affectif ne sort pas de la momentanéité du discours, qu'il n'appartient pas à la langue, mais au discours seulement » - cité par Monneret (ibid. : 30). 
11. Sur la conception historique de la ponctuation et sa théorisation, voir Jaffré (1991), Anis (2004), Serça (2004), Rault (2014a, b). On est donc en apparence en contradiction avec l'évolution actuelle de la recherche, ainsi présentée par J. Rault (2014b: 10), Dürrenmatt (2015) : « De la grammaticalisation à la "structure sémantique" d'Ivan Fonagy et au "signifié en langue" de Jacqueline Authier-Revuz se dessine un large mouvement d'inclusion [de la ponctuation] dans le système de la langue, sous forme de sémiotisation. » La question est de savoir ce que l'on entend désormais par grammaire voir conclusion, infra.

12. On se souvient que l'une des toutes premières études d'inspiration linguistique de la ponctuation en français, procurée par H. Sensine en 1930 est publiée « avec soixante dictées-exercices dans le texte » (sic).

13. Cette idée présentée très souvent par H. Meschonnic dans ses différents travaux, a été reprise par I. Serça (2012).

14. Voir la mise au point de J. Rault (2014b:9) : « C'est bien en se dégageant progressivement du phonocentrisme qu'une véritable étude de la ponctuation a pu advenir, en posant notamment, depuis quelques années, les enjeux en termes sémiotiques. »

15. Voir le classement de "perspective stylistique » proposé par J. Popin (1998: 82) : les « signes purs » (sic) que sont la virgule, le point-virgule, le point et le point d'interrogation ; les « signes par évocation » que sont les points de suspension et le point d'exclamation; les « signes de l'énonciation » que sont les deux-points, les guillemets, les parenthèses et le tiret.

16. Voir la conclusion de J. Anis (2004 : 10) qui cite P. Dahlet : « Loin de se cantonner aux signes d'énonciation, la dimension énonciative gagne l'ensemble de la ponctuation, et singulièrement par l'orientation pragmatique qu'elle indique. » 17. Le travail sur des journaux intimes pour une étude de la ponctuation de l'écrit est particulièrement satisfaisant pour un stylisticien. Parce que ce genre poétique est d'abord une pratique de l'extériorisation des sentiments devenue représentation d'une autoénonciation : le scripteur use de codes graphiques et de choix textuels qui sont au service d'une lisibilité et d'une transcriptibilité de son émotion dans un propos qui n'est rien d'autre qu'une expressivité émotive devenue expression formelle. L'idée d'une oralisation de l'énonciation n'est pas un contresens artificiel dans ce contexte : la dramatisation des unités de ponctuation les plus diverses dans le texte a pour effet de suggérer puissamment un effet de voix (intérieure ?) identificatrice qui régule le régime stylistique de l'ensemble. Un journal intime se parle autant qu'il s'écrit : on ne dirait pas du tout cela du roman, par exemple.

18. L. Bloy, Journal, Paris, Robert-Laffont (« Bouquins »), 1999, t. I, p. 137 (mars 1895).

19. Ibid., p. 72 (novembre 1893).

20. Ibid., p. 78 (mars 1894).

21. Ibid., p. 365 (décembre 1900).

22. Ibid., p. 83 (avril 1894).

23. Ibid., p. 168 (novembre 1895).

24. Ibid., p. 254 (avril 1899).

25. Exemple de ce que L. Rosier (1998 : 361) appelle « les capitales ton de voix ou la vocifération ».

26. Dans Béatrix (1839), Balzac note finement, à propos de la lettre fort exaltée d'un jeune héros éminemment romantique : « il y procédait par phrases exclamatives; il y avait beaucoup de ces points prodigués par la littérature moderne dans les passages dangereux, 
comme des planches offertes à l'imagination du lecteur pour lui faire franchir les abîmes » - in Balzac, La Comédie humaine, Paris, Gallimard (« La Pléiade »), 1976-1981, t. II, p. 784. Pour une contextualisation historique en période romantique de la question de l'expressivité et des ambiguïtés qu'elle découvre en discours, voir Dürrenmatt (2001).

27. Op. cit., p. 81 (mars 1894).

28. Ibid., p. 231 (septembre 1898).

29. Sur la linguistique générale de l'exclamation en français, en particulier ses réalisations syntaxiques, voir Bacha (2000).

30. Voir la conclusion : «Alors nous comprîmes que le Sauveur, qui avait voulu ce témoignage, était infiniment éloigné de nous redemander nos âmes, qu'il nous sollicitait seulement de les lui prêter un peu en vue d'accomplir quelque chose qui avait "manqué à sa Passion". »-ibid., p. 232.

31. Sur la ponctuation textuelle des paragraphes narratifs, voir Arabyan (1994); sur la richesse de l'exploitation littéraire de cette ressource typographique, voir Dürrenmatt (1998 : 95-111).

32. Qui se réalise exemplairement en langue dans tous les phénomènes de détachement. On reste très surpris de constater que les linguistes qui travaillent sur la question du détachement (apposition, construction détachée, etc.) n'envisagent jamais leur objet en termes de ponctuation. Les Actes du colloque de Nancy en 2006 consacré aux « linguistiques du détachement » (Apothéloz et al., 2009) n'ont pas un mot pour la ponctuation, à l'exception de A. Kuyumcuyan et d'une autre étude (M. Colas-Blaise), mais sur corpus littéraire (l'usage des tirets chez Gracq) et par là de coloration plus stylistique que linguistique. On ne parle pas tant de ponctuation typographique, point de départ sinon objet de l'étude d'A. Kuyumcuyan (les compléments d'objet après le point), que de ponctuation textuelle générale : la question, par exemple, des relatives ou des épithètes en construction détachée relève entièrement d'une pensée de la ponctuation. Qui ne saurait être ramenée aux « facteurs stylistiques » ou à la « valeur expressive » des grammaires citées ci-dessus. Quand les linguistes travaillent sur la ponctuation de l'écrit ( in Defays et al. [1998], par exemple), il s'agit pour eux de se concentrer strictement sur ce que L. Védénina (1989) avait appelé la " pertinence de la présentation typographique » imprimée. Une extension de la notion aux autres formes d'expression réalisées par le détachement serait utile.

\section{RÉSUMÉS}

Peut-on envisager une grammaire de l'expressivité posée et représentée par l'énonciation dans les manifestations textuelles de ce que l'on appelle "ponctuation»? Oui, s'il s'agit d'une grammaire stylistique, c'est-à-dire une grammaire du singulier qui s'attache à décrire tout ce qu'un sujet individuel peut faire passer de lui-même par la langue dans un énoncé et qui travaille sur la valeur comme performance dans ce vaste régulateur discursif qu'est le style. Dans cette perspective, la ponctuation ne peut que jouer un rôle majeur, se confondant entièrement avec l'énonciation, phénomène induit et produit par l'historicité fondatrice d'un sujet s'exprimant 
dans le temps de son discours : loin d'être un complément d'information, elle découvrirait même la base de la diction textuelle sans laquelle il n'y aurait pas de style.

Can one consider a grammar of the expressivity posed and represented by the stating in the textual demonstrations of what one calls "punctuation"? Yes, if it is about a stylistic grammar, i.e. a grammar of the singular which attempts to describe all that an individual subject can make pass from itself by the language in a statement and which works on the value as performance in this vast discursive regulator which is the style. From this point of view, the punctuation can only play one main function, merging entirely with the stating, phenomenon induced and produced by historicity founder of a subject being expressed in the time of its speech: far from being a further information, she would discover even the base of the textual diction without which there would be no style.

INDEX

Mots-clés : ponctuation à valeur expressive, ponctuation à valeur sémantique, grammaire, style

\section{AUTEUR}

\section{ÉRIC BORDAS}

École Normale Supérieure de Lyon - UMR 5611 LIRE 